\title{
REVISTAMARACANAN
}

Nota de Pesquisa

\section{Consideraciones en torno al contacto entre lenguas y el cambio lingüístico. Repensando el bilingüismo sumerio- acadio del tercer y segundo milenio a. C. ${ }^{1}$}

\author{
Considerações sobre o contato entre línguas e mudança linguística. \\ Repensando o bilingualismo sumério-acádio no terceiro e segundo milénio a.
}

C.

\author{
Considerations about the Contact among Languages and the Language \\ Change. Rethinking Sumerian-Akkadian Bilingualism during the Third and \\ Second Millennium B.C.
}

Rodrigo Cabrera

Universidad de Buenos Aires cabrera.pertusatti@gmail.com

\begin{abstract}
Resumen: En la presente investigación, teniendo en cuenta el fenómeno de "cambio lingüístico", estudiaremos la manera en que dos lenguas de la Baja Mesopotamia, el sumerio y el acadio, se vincularon e influenciaron mutuamente entre el tercer y el segundo milenio a.C. a partir de préstamos semánticos y sintácticos. Asimismo, consideraremos las aproximaciones etnográficas y arqueológicas efectuadas por diversos estudiosos para pensar la manera en que ambas lenguas se enlazaron. Finalmente, reflexionaremos sobre el uso de topónimos demarcadores de alteridades demográficas, los cuales evidencian, como en el caso de los mar-tu, la existencia de poblaciones extranjeras con hábitos señalados como inferiores, pero que a posteriori consiguen la hegemonía sociopolítica en la región.
\end{abstract}

Palabras clave: Cambio lingüístico; Sumerio; Acadio; Mesopotamia; Mar-tu.

Resumo: Na presente pesquisa, tendo em conta o fenômeno da "mudança linguística", estudaremos como as duas línguas da Baixa Mesopotâmia, o sumério e o acádio, estiveram ligadas e influenciaram-se mutuamente entre o terceiro e segundo milênios a. C. a partir de empréstimos semânticos e sintáticos. Além disso, consideramos as abordagens etnográficas e arqueológicas feitas por vários estudiosos que pensaram sobre como ambas as línguas estiveram ligadas. Finalmente, vamos refletir sobre o uso de topônimos demarcadores de alteridades demográficas que mostram, como no caso de mar-tu, a existência de populações estrangeiras com hábitos marcados como inferiores, mas que, posteriormente, obtiveram a hegemonia político-social na região.

Palavras-chave: Mudança linguística; Sumério; Acádio; Mesopotâmia; Mar-tu.

Abstract: In this paper, we will consider the "language change" phenomenon to study the way in which two languages of Lower Mesopotamia, Sumerian and Akkadian, were linked and influenced each other between the third and second millennium BC from semantic and syntactic loans. Likewise, we will consider the ethnographic and archaeological approaches made by different scholars to think about the way in which both languages were linked. Finally, we will reflect on the use of place-names that points demographic alterity, which show, as in the case of the mar-tu, the existence of foreign populations with habits designated as inferiors, but which subsequently achieve socio-political hegemony in the region.

Keywords: Language change; Sumerian; Akkadian; Mesopotamia; Aar-tu. 


\section{Aspectos generales del contacto entre lenguas y el cambio lingüístico}

La reconstrucción de los procesos socio-históricos del Próximo Oriente antiguo implica un diálogo interdisciplinario entre los estudios filológicos y arqueológicos de forma respectiva. Por consiguiente, el cambio histórico puede ser aprehendido a partir de un análisis riguroso de fuentes epigráficas y de su posterior (o anterior) confrontación con el registro material dejado por las sociedades antiguas.

En el presente artículo, nos enfocaremos en los factores del "cambio lingüístico" y de qué manera el mismo puede ser reconocido e individualizado en el registro escrito. Para ello, examinaremos los fenómenos de cambio lingüístico que se dieron en Mesopotamia aproximadamente entre el 3000 y el 600 a.C., en cuyo momento se produjo la predominancia de dos grandes grupos lingüísticos: el sumerio y el acadio.

En relación al primero, se ha hablado de la "cuestión" o "problema" sumerio, dado su aislamiento y supuesta pureza lingüística. Dicho debate continúa hasta la actualidad y, a propósito, en una de las Rencontre Assyriologique Internationale (RAI) celebrada en 2002 en Leiden, ${ }^{2}$ la discusión fue retomada por algunos estudiosos como G. Rubio y G. Whittaker.

Para ilustrar la controversia ante el fenómeno del "cambio lingüístico", debemos tener en cuenta, por ejemplo, problemáticas asociadas a la circulación poblacional y/o de transformaciones en la coyuntura política, que han influenciado en el proceso a partir del cual una lengua se impone sobre otra/s y adquiere, así, preeminencia en una región específica. En este sentido, las migraciones de pueblos semi-nómades hablantes de lenguas semitas a la región sur de Mesopotamia es un proceso reconocible desde el tercer milenio a.C., cuando aparecen mencionados en la documentación a través de una diversidad de topónimos y gentilicios. Este es el caso de los mar-tu sobre el que volveremos más adelante, los cuales, en una primera etapa, emplearon el sumerio en la administración y en la literatura y, luego, utilizaron el acadio.

A fines de siglo XIX, H. Schuchardt sostenía la inexistencia de un idioma que se encontrara libre de cualquier influencia externa. ${ }^{3}$ Años después, U. Weinreich postulaba que el "contacto" debe entenderse como la modalidad por excelencia para que se produzca el cambio lingüístico en cuanto al léxico, la fonología, la morfología y/o la sintaxis, entre otros ${ }^{4}$. De acuerdo a P. Epps, J. Huehnergard y N. Pat-El, "los lingüistas ven en el contacto uno de los principales

\footnotetext{
${ }^{1}$ La presente investigación está financiada por una beca de doctorado otorgada por el Consejo Nacional de Investigaciones Científicas y Técnicas (CONICET, Argentina).

${ }^{2}$ Cf. VAN SOLDT, Wilfred H. (Ed.). Ethnicity in Ancient Mesopotamia. Papers Read at the 48th Recontre Assyriologique Internationale, Leiden, 1-4 July 2002. Leiden: Nederlands Instituut voor het Nabije Oosten, 2005.

${ }^{3}$ SCHUCHARDT, Hugo Ernst Mario. Dem Herrn Franz von Miklosich zum 20. November 1883: Slawodeutsches und Slavo-italienisches. Graz: Leuschner and Lubensky, 1884, p. 5.

${ }^{4}$ WEINREICH, Uriel. Languages in Contact: Languages in Contact: Findings and Problems. New York: Linguistic Circle of New York, 1953.
} 
mecanismos para el cambio idiomático, con algunos que van tan lejos como sugerir que el contacto es el principal catalizador para el cambio". ${ }^{5}$

En cuanto al contexto de contacto, no podemos postular la existencia de cambio a partir de estructuras gramaticales similares, puesto que si bien se da una correlación entre similitud estructural y cambio estructural, puede ocurrir lo contrario. ${ }^{6}$ Asimismo, tanto la predominancia del contexto social o de los factores lingüísticos no consideran uno de los puntos centrales a partir del cual se dan las semejanzas estructurales y/o el contacto entre poblaciones: la estrecha relación genética. ${ }^{7}$

La lingüística diacrónica se presenta como la disciplina que apunta al análisis del cambio de las lenguas a través del tiempo $y$, en muchos casos, sus alcances son cotejados con los resultados ofrecidos por otras disciplinas como la historia, la arqueología y/o la genética. De esta manera, se busca la reconstrucción del mapa lingüístico del mundo, atendiendo a las variaciones epocales que sufren las lenguas. A propósito, las lenguas "separadas" de forma reciente pueden ubicarse en la misma zona geográfica $y$, por ello, sus hablantes pueden continuar en contacto permanente. $^{8}$

Por ejemplo, en cuanto a las lenguas de la región sirio-palestinense, podríamos preguntarnos en el caso del hebreo bíblico y el arameo cuál de las dos sería la "original" y cuál la "foránea", así como también, cuándo se habría producido el contacto entre ambas. Así, en el hebreo bíblico, el masculino plural se escribe con el sufijo -īm y, en arameo, con -īn. No obstante, en el contexto sociolingüístico del hebreo post-bíblico, ambos sufijos ocurren de manera indistinta, dado el bilingüismo de sus hablantes ${ }^{9}$. De este modo, podríamos indagar en el caso del arameo y el hebreo bíblico, en cuanto lenguas semitas, por la existencia de una protolengua de la cual se dividen y desarrollan. Asimismo, podríamos explorar de qué manera entre lenguas hermanas y dialectos, que residen en áreas geográficas contiguas, se produce el contacto regular y la transferencia entre sus respectivas comunidades de hablantes.

En síntesis, el error más usual, a pesar del conocimiento y estudio del término Sprachbund, es basarse en el estudio de léxicos estáticos y, además, el contacto entre dialectos en un estadio temprano de la diferenciación lingüística puede conducir a la carencia de divisiones binarias claras. ${ }^{10}$

\section{Contacto y cambio lingüístico a partir del análisis del sumerio y el acadio en perspectiva diacrónica}

\footnotetext{
${ }^{5}$ EPPS, Patience; HUEHNERGARD, Johnand \& PAT-EL, Na'ama. Introduction: Contact among Genetically Related Languages. Journal of Language Contact, v. 6, n. 2, p. 209-219, 2013, p. 209.

6 HAIG, Geoffrey. Linguistic Diffusion in Present-Day East Anatolia: From Top to Bottom. En: AIKHENVALD, Alexandra; DIXON, Robert (eds.). Areal Diffusion and Genetic Inheritance: Problems in Comparative Linguistics. Oxford: Oxford University Press, 2001, p. 195-224.

7 EPPS et al., Op. Cit., p. 210.

${ }^{8}$ Ibídem, p. 211.

${ }^{9}$ EPPS, et al. Introduction... Op. cit., p. 211.

${ }^{10}$ Ibídem, p. 212.
} 
En el caso del sumerio y el acadio, dos lenguas no emparentadas, reconocemos una serie de préstamos y afluencias recíprocas desde el Dinástico Temprano, cuando ambas comienzan a convivir en la Baja Mesopotamia (Cuadro 1). De acuerdo con G. Zólyomi, el acadio se desarrolló bajo la influencia y empleando préstamos del sumerio, cuyos rasgos se evidencian en el uso de topónimos y en la distribución de textos y tipologías textuales. ${ }^{11}$

\begin{tabular}{|c|c|c|c|}
\hline \multicolumn{4}{|c|}{ CUADRO 1} \\
\hline \multicolumn{3}{|c|}{ Períodos de la Baja Mesopotamia } & CRONOLOGÍA \\
\hline \multirow{4}{*}{$\begin{array}{l}\text { Dinástico } \\
\text { Temprano }\end{array}$} & \multicolumn{2}{|c|}{$\mathbf{I}$} & $\begin{array}{l}2900-2750 \\
\text { a.C. }\end{array}$ \\
\hline & \multicolumn{2}{|c|}{ II } & $\begin{array}{l}2750-2600 \\
\text { a.C. }\end{array}$ \\
\hline & \multirow[t]{2}{*}{ III } & A & $\begin{array}{l}2600-2500 \\
\text { a.C. }\end{array}$ \\
\hline & & B & $\begin{array}{l}2500-2350 \\
\text { a.C. }\end{array}$ \\
\hline \multicolumn{3}{|c|}{ Paleo-acadio o sargónico } & $\begin{array}{l}2350-2200 \\
\text { a.C. }\end{array}$ \\
\hline \multicolumn{3}{|c|}{ Lagaš II } & $\begin{array}{l}2200-2100 \\
\text { a.C. }\end{array}$ \\
\hline \multicolumn{3}{|c|}{ AMORROS Ur III (neo-sumerio) } & $\begin{array}{l}2100-2000 \\
\text { a.C. }\end{array}$ \\
\hline \multirow{2}{*}{\multicolumn{2}{|c|}{ Paleo-babilónico }} & Isin & \\
\hline & & $\begin{array}{c}\text { Larsa } \\
\text { Babilonia }\end{array}$ & $\begin{array}{l}2000-1600 \\
\text { a.C. }\end{array}$ \\
\hline \multicolumn{3}{|c|}{ Meso-babilónico } & $\begin{array}{l}1600-1000 \\
\text { a.C. }\end{array}$ \\
\hline \multicolumn{3}{|c|}{ Neo-babilónico } & 1000-539 a.C. \\
\hline
\end{tabular}

Cuadro 1: Cronologías y períodos de la Baja la Mesopotamia

La influencia más notable del sumerio en el acadio se da en el orden estructural adoptado por ambas lenguas, el cual consta de sujeto-objeto-verbo. En cambio, el semita occidental mantuvo el verbo inicial como herencia del proto-semítico. Otro de los préstamos más notables del sumerio en el acadio tiene que ver con el conjunto lexical, puesto que los vocablos sumerios constituyen el $7 \%$ del vocabulario acadio, ${ }^{12}$ siendo en su mayoría sustantivos.

Asimismo, en el acadio, bajo la influencia lingüística del sumerio, se produce la fusión y pérdida de cinco consonantes guturales proto-semitas reconstruidas: /\%/, /h/, /h/, /؟/ y /g்/, puesto que el sumerio carecía de tales fonemas. ${ }^{13}$ Otro rasgo gramatical desarrollado bajo la

\footnotetext{
11 ZÓLYOMI, Gábor. Akkadian and Sumerian Language Contact. En: WENINGER, Stefan. (Ed.). The Semitic Languages: An International handbook. Berlin-New York: Mouton de Gruyter, 2011, p. 396-404 12 EDZARD, Dietz-Otto. Sumerian Grammar. Leiden-Boston: Brill, 2003, p. 178.

13 Tres de estos $(/ \mathrm{h} /, / \varsigma /, / \dot{g} /)$ causaron la marcación de una [a] continua a [e]. Esta [e] alofónica se convirtió en fonética, indicado que se mantuvo después de la pérdida de las guturales. El fonema /e/ se presenta como diferenciador de funciones de guturales perdidas. Por otro lado, se produjo la armonía vocálica del dialecto babilónico del acadio.
} 
influencia del sumerio por el acadio es el uso del cislocativo o ventivo marcado por los sufijos $m,{ }^{14}$-am, -nim en el último y por el prefijo $m(V)$ en el primero. En cuanto al sistema pronominal, los dialectos acadio sargónico y paleo-babilónico poseen un número más elevado de distinciones de caso, si lo comparamos con el paleo-asirio y el proto-semita.

Además, el sistema modal del acadio admite dos: la partícula $/ \bar{u}$ - y el prefijo verbal $I V-$, la primera como aseverativa, delante de formas sin prefijos pronominales, $y$, la otra, como marca modalidad deóntica. Posteriormente, se produce el solapamiento de ambas por la influencia del sumerio, puesto que el prefijo modal $h \mathrm{~V}$ - abarca tanto $I V$ - como $I \bar{u}-.^{15}$

Por otro lado, en relación al uso del estativo, en acadio, el mismo se presenta como una construcción en la que un adjetivo (verbal) o un sustantivo forma un predicado con una cópula pronominal. Varias características de esta construcción se han conectado con la influencia del sumerio que contiene predicados no verbales con una cópula verbal.

En relación a los tiempos verbales, el sistema de tiempos (tenses) en acadio y sumerio presenta semejanzas: el presente-futuro en sumerio (formas marû) es similar al acadio iparrVs, que señala una acción simultánea o posterior; y el pretérito en sumerio (formas hamțu) corresponde al acadio iprVs, que denota una acción relativa anterior a un punto de referencia dado por el contexto. ${ }^{16}$

Otro de los rasgos gramaticales, tomados por el acadio del sumerio, tiene que ver con la construcción de subordinadas, las cuales aparecen en acadio con los sufijos -u/-ni/-ūni mientras, en sumerio, se usan para las formas finitas el sufijo $/\left(^{\prime}\right) a /$.

Por otra parte, no sabemos prácticamente nada sobre el contacto en la etapa previa al Dinástico Temprano, es decir, durante el Ilamado período Uruk Tardío, del cual nos ha quedado una prolífica colección de tablillas administrativas, que dan cuenta del sistema socioeconómico del aluvión mesopotámico para la mencionada época.

Como señalábamos más arriba, en la $R A I$ del 2002, se retomó un antiguo debate sobre la lengua sumeria y su controvertido origen, que ya en el 1944 B. Landsberger se había propuesto estudiar. Este último planteó la existencia de un hipotético sustrato pre-sumerio, al cual abonaron otros estudiosos de la talla de I. Gelb, A. L. Oppenheim, A. Salonen, I. Diakonoff y J. Bauer, y al que se le otorgó una serie de características arqueológicas, físico-antropológicas, literarias, lingüísticas, grafemáticas, étnicas y toponímicas. ${ }^{17}$ La construcción intelectual de un sustrato pre-sumerio para Landsberger señaló la definición de una delimitación lingüística semejante al sustrato pre-indoiranio, aunque en este último sí puede reconocerse un contexto

\footnotetext{
${ }^{14}$ El cislocatio o ventivo indica movimiento hacia un centro deíctico: puede ser la localización del acto de habla o de alguno de los participantes del acto de habla. También, podría funcionar como la primera persona del singular.

${ }^{15}$ El solapamiento ocurre por la existencia de dos formas para marcar la negación deóntica: en acadio, a través del prohibitivo y el vetitivo; en el sumerio con las formas ba ra - y na -

${ }^{16}$ A propósito, el perfecto en acadio (iptarVs) se desarrolla a partir del pretérito del Gt-stem, formado con un infijo $t$-. El mismo es una innovación acadia o semita oriental, que se piensa como una detransitivization, conectada con el prefijo sumerio b a - .

${ }^{17}$ RUBIO, Gonzalo. On the Linguistic Landscape of Early Mesopotamia. En: VAN SOLDT, Wilfred H. (Ed.). Ethnicity in Ancient Mesopotamia. Papers Read at the 48th Recontre Assyriologique Internationale, Leiden, 1-4 July 2002. Leiden: Nederlands Instituut voor het Nabije Oosten, 2005, p. 317.
} 
lingüístico, cultural e histórico mejor definido. ${ }^{18}$ Según él, el criterio para señalar la presencia de vocablos no sumerios era básicamente fonotáctico; esto es, mientras el sumerio tenía una terminología preferentemente monosilábica, los préstamos eran en su mayoría polisilábicos con grupos consonánticos en posición medial y sin etimologías sumerias. ${ }^{19}$ A propósito, Rubio plantea la inexistencia de un sustrato en el sentido que lo propone Landsberger, pero no logra terminar de rebatir la hipótesis inicial de éste.

De acuerdo a Whittaker, quien señala las falencias de la perspectiva de Rubio en un artículo publicado en 1999 para refutar a Landsberger, ${ }^{20}$ no se ha conseguido superar la hipótesis inicial planteada por este último. ${ }^{21}$ Asimismo, Whittaker partiendo de la pregunta inicial sobre el origen de los sumerios y por su penetración primigenia en el sur de Mesopotamia, afirma que es imposible "identificar un grupo étnico sobre la base de la arqueología y la historia del arte únicamente -al menos, la evidencia toma la forma de documentos escritos tales como inscripciones y registros administrativos. En el pasado, la continuidad en el registro arqueológico era vista frecuentemente como un indicador de continuidad étnica, así como la interrupción era vista como un indicador del arribo de uno o varios pueblos nuevos". ${ }^{22}$

Por lo tanto, es absolutamente arriesgado postular la continuidad étnica a partir del análisis arqueológico, pero también es de igual modo peligroso proponerlo cuando analizamos el registro epigráfico, aunque éste no muestre rupturas claras. Como sostiene Whittaker, estamos en presencia de "dos comunidades de hablantes que comparten una cultura común a la cual han contribuido substancialmente ambas. La información idiomática es fundamental para solucionar estas cuestiones". ${ }^{23}$ De forma semejante, G. Zólyomi sostiene para el período Dinástico Temprano en adelante, más allá de la cantidad de préstamos sumerios recibidos por el acadio el cual se habría desarrollado a partir del anterior-, la existencia de un bilingüismo prolongado a partir del desarrollo de cada lengua. ${ }^{24}$

En síntesis, debemos comenzar con el estudio del lenguaje de los documentos escritos, de su contenido, del análisis lexical, de los nombres propios y de los sistemas de escritura para poder comprender los fenómenos de cambio lingüístico en la Baja Mesopotamia.

Otros posicionamientos sobre la "problemática/cuestión sumeria" y su punto de despegue en el aluvión mesopotámico han recurrido al análisis etnológico haciendo un uso y abuso especulativo, como es el caso de J. Bottéro. Para este estudioso, remontándose al ambiguo paisaje mono- o multiétnico de los períodos Ubaid o Uruk, los sumerios penetraron en Mesopotamia por el sureste y se mezclaron con una primigenia población de "nativos" y con otra de origen semita, proveniente de las fronteras norte y este del gran desierto sirio-árabe, que

\footnotetext{
18 RUBIO, Gonzalo. On the Linguistic... Op. cit., p. 318.

19 Ibídem, p. 317.

${ }^{20}$ RUBIO, Gonzalo. On the Alleged 'Pre-Sumerian Substratum'. Journal of Cuneiform Studies, v. 51, 1999 , p. $1-16$.

${ }^{21}$ WHITTAKER, Gordon. The Sumerian Question: Reviewing the Issues. En: VAN SOLDT, Wilfred H. (Ed.). Ethnicity in Ancient Mesopotamia. Papers Read at the 48th Recontre Assyriologique Internationale, Leiden, 1-4 July 2002. Leiden: Nederlands Instituut voor het Nabije Oosten, 2005, p. 409.

22 Op. cit., p. 410.

23 Ibídem, p. 410.

${ }^{24}$ ZÓLYOMI, Gábor. Akkadian and Sumerian Language... Op. cit., p. 397-398.
} 
abandonó su vida semi-nómade y se infiltró en los establecimientos de los sedentarios. Por su lado, H. Nissen, tomando el análisis de patrones de asentamiento, afirma que los sumerios llegaron a Mesopotamia con probabilidad en el Uruk Tardío.

En conclusión, se evidencia un uso excesivo de la perspectiva etnográfica para abordar la cuestión sumeria no sólo en el análisis del registro arqueológico, sino también del epigráfico y, por consiguiente, los estudios etno-demográficos y la dialéctica nómade-sedentario, que estuvieron en boga en los '70 en el ámbito asiriológico, han pretendido explicar equívocamente los cambios lingüísticos ocurridos en Mesopotamia.

\section{Los mar-tu y el cambio lingüístico: más allá del bilingüismo sumerio- acadio}

Volviendo al Cuadro 1, en Mesopotamia, tenemos la convivencia o bilingüismo desde el Dinástico Temprano del sumerio y el acadio. El primero desapareció luego del período paleobabilónico, cuando dejó de emplearse tanto en la literatura como en la administración. Cabe recordar que si bien el sumerio había dejado de utilizarse con anterioridad durante el período sargónico, la dinastía de Ur III, heredera de la gloriosa Akkad, reutilizó diversos tópicos del Dinástico Temprano e incluso reintrodujo el uso del sumerio en la administración. A propósito, sus dos primeros reyes llevaron nombres sumerios (Ur-Nammu y Šulgi), mientras los otros tres emplearon nombres semitas (Amar-Suen, Šu-Suen y Ibbi-Suen). ${ }^{25}$ De acuerdo a Rubio, aunque la lengua empleada en la época del reinado de Šulgi -vale recordar que estuvo en el poder alrededor de 48 años- fuera el sumerio, la lengua nativa del monarca tal vez haya sido el acadio. $^{26}$

La era de mayor desarrollo de la literatura sumeria corresponde con la aparición en escena de nuevas configuraciones políticas y también socioculturales en la región meridional de Mesopotamia. Por cierto, durante los períodos de Ur III y paleo-babilónico, en el sumerio, se da un salto cualitativo en cuanto a la difusión de nuevas tipologías textuales, que presentan, además, una serie de variaciones en los planos fonológico y sintáctico. ${ }^{27}$ Esto se debe a que los manuscritos recuperados provienen de contextos educativos y constituyen el acervo pertenecientes a las escuelas de escriba mesopotámicas, donde los concurrentes -los cuales no eran hablantes nativos de sumerio- iban a aprender la lengua y el sistema de escritura

25 RUBIO, Gonzalo. Sumerian Literature. En: EHRLICH, Carl S. (Ed). From an Antique Land. An Introduction to Ancient Near Eastern Literature. Lanham-Boulder-New York-Toronto-Plymouth: Rowman \& Littlefield Publishers, Inc., 2009, p. 17-18.

${ }^{26}$ RUBIO, Gonzalo. Šulgi and the Death of Sumerian. En: MICHALOWSKI, Piotr; VELDHUIS, Niek. (Eds.). Approaches to Sumerian Literature: Studies in Honour of Stip H.L.J. Vanstiphout. Leiden: Brill, 2006, p. 167-180.

27 BLACK, Jeremy \& ZÓLYOMI, Gábor. Introduction to the Study of Sumerian. En: EBELING, Jarle; CUNNINGHAM, Graham. (Eds.). Analyzing Literary Sumerian: Corpus-based Approaches. London: Equinox, 2007, p. 1. 
cuneiforme, pero contando, en muchos casos, con patrones gramaticales disímiles a los del sumerio clásico.

Por ende, el bilingüismo del sur mesopotámico, permitía que los escribas no hablantes de sumerio compilaran toda la tradición cultural anterior y la readaptaran también al nuevo clima social de la época. Asimismo, es sumamente difícil, en ciertos casos, distinguir un error de una variación textual $y$, por tal motivo, resulta compleja la manera en cómo se reproducía una ortografía correcta 0 estandarización. ${ }^{28}$ En este sentido, la comparación entre textos administrativos y textos literarios, puesto que manejan dos registros de lengua diferentes, es del todo oportuna y necesaria. Por ejemplo, en los textos literarios (himnos y plegarias) del rey Šulgi, se emplea una ortografía arcaica e incluso divergente para señalar que se están aplicando cánones textuales distintos a los que se dan en los textos administrativos.

Además, a partir del análisis de las tablillas cuneiformes recuperadas, el uso de ortografía divergente se evidencia en cuanto a la fonética, puesto que se escriben a partir del nivel del lenguaje hablado y los sonidos se reproducen de acuerdo a las necesidades de pronunciación de los aprendices, efectuándose un reemplazo de escritura logográfica con signos cortos o comunes -como ocurre en el dialecto Emesal. ${ }^{29}$

Otra cuestión a tener en cuenta es el momento en el que ocurre la muerte del sumerio. A propósito, debemos pensar que se trata de un proceso gradual, que quizás se dé como consecuencia del contacto lingüístico, aunque su uso se haya prolongado en la literatura y la administración hasta fines del período paleo-babilónico. Como postulan Black y Zólyomi, ${ }^{30}$ existía un empleo vernacular del sumerio en cartas o documentos legales y otro formal en textos administrativos, que parecieran contener una gramática propia. El uso informal o vernacular del sumerio se prolongó hasta la época de Lipit-Ištar (1934-1924 a.C.). Luego del 1800 a.C., el sumerio es registrado esporádicamente en inscripciones reales y en algunos documentos legales, como se da en Nippur hasta 1730 a.C. ${ }^{31}$

En muchos casos, el registro formal de los textos literarios se utiliza abiertamente en las inscripciones reales y, asimismo, la literatura aparece como una evocación del registro oral en diversos manuscritos.

De modo que, así como el acadio se fue adaptando y, además, adoptando características no sólo gramaticales del sumerio, los escribas hablantes de acadio, que estudiaban y empleaban el sumerio en diversas tipologías textuales entre los períodos neo-sumerio y paleo-babilónico, también contribuyeron a estimular ciertos cambios en el mismo. Por ejemplo, ciertas distinciones gramaticales propias del sumerio comenzaron a desaparecer: el uso de los posesivos -a ni y bi (para los géneros humano y no humano respectivamente) ya no siguieron la lógica diferencial precedente; el empleo de formas causativas con -/ra/ (humano) y -/e/ o -/a / (no

\footnotetext{
${ }^{28}$ BLACK, Jeremy \& ZÓLYOMI, Gábor. Introduction to the... Op. cit., p. 4.

29 El Emesal, literalmente "lengua de mujer" en sumerio, corresponde a un registro de la lengua empleado en literatura. Presenta un grado de alteración fonológica elevado, que quizás esté originado en el lenguaje hablado bajo la influencia del acadio.

30 BLACK, Jeremy \& ZÓLYOMI, Gábor. Introduction to... Op. cit., passim.

${ }^{31}$ Ibídem, p. 6.
} 
humanos), desde Ur III en adelante, después del genitivo -/ak/ se hicieron frecuentes; algunas distinciones en casos cambiaron a partir de la influencia del acadio como sucedió con el locativo; se produjo la interferencia de estructuras (empleo del genitivo indefinido, formas verbales causativas con / $\mathrm{b}$ / / y / $\mathrm{n}$ / / similares a formas verbales acadias, variación del régimen verbal de ergativo-absolutivo, formas precativas con la construcción en hamțu); se produjo una transferencia de reglas gramaticales (pérdida de mimación, etc.).

En efecto, el sumerio, que habría funcionado como el latín en la Edad Media, cayó en desuso luego del período paleo-babilónico, cuando finalmente el acadio se convirtió en la lengua preponderante en todos los planos discursivos. Por otro lado, durante Ur III, reconocemos la preeminencia de otra problemática en la región: la presencia del término mar-tu o amorreo, un concepto polisémico, que designaba, entre otras cosas, a las poblaciones semi-nómades del Occidente de Mesopotamia. Además, el vocablo aludía a una divinidad de origen semita que aparece en algunas fuentes como El casamiento de Martu. ${ }^{32}$ No es arbitrario, que la narración, si bien es recogida en copias paleo-babilónicas de las escuelas de escribas de Ur y Nippur, retome una problemática de fines del tercer milenio a.C., puesto que la infiltración de poblaciones pastoriles semi-nómades de Occidente era un acontecimiento habitual y hasta económicamente necesario. Al mismo tiempo, el relato describe los intercambios culturales entre las poblaciones mesopotámicas y explica -o justifica- la fusión de los grupos poblacionales, puesto que recoge las tradiciones de los amorreos, que se hicieron con el poder en época paleo-babilónica.

El casamiento de Martu no sólo señala la existencia de alteridades demográficas con hábitos que se consideraban inferiores, sino que además describe a los hablantes de una lengua diferente a la empleada para escribir el relato: la lengua de los amorreos. De acuerdo con la descripción, que en ningún caso pretende ser etnográfica, sino más bien marcar las tensiones sociopolíticas de la segunda mitad del tercer milenio a.C., se observa la hegemonía del sumerio como lengua de las ciudades-estado mesopotámicas, ya que es la que nomina y domina en simultáneo.

Si nos detenemos en la aparición del vocablo mar-tu en la documentación administrativa mesopotámica, observamos que ya son mencionados como población durante Lagaš II. A propósito, la tablilla MVN 06249 (BDTNS 20414) registra: 2 dug geštin martu $[\ldots] U_{3}-r i_{2}-b a-a d$, esto es, se envían dos jarras de vino de parte de un mar-tu. Durante Ur III, las inscripciones que recogen esta nominación son diversas, registrándose el término unas 3816 veces. Por ejemplo, la tablilla ITT 506744 (BDTNS 005608) (del mes 7 del año 11 del reinado de Šulgi) afirma que: $15 \mathrm{gada} u s_{2}(\ldots) \mathrm{mar}-\mathrm{tu} \mathrm{i}_{3}-\mathrm{de}_{6}$, es decir, que los mar-tu llevaron 15 trajes de lino. Por consiguiente, las relaciones e intercambios económicos y políticos entre las ciudades-estado sumerias y los grupos semi-nómades de la región Siria fueron habituales. Asimismo, los préstamos lingüísticos incrustados en este juego de relaciones sociales también habrían sido constantes.

\footnotetext{
32 BLACK, Jeremy et al. The Electronic Text Corpus of Sumerian Literature. Oxford: Oriental Institute of the University of Oxford, 1998, c.1.7.1 y t.1.7.1. Disponible en:<http://www-etcsl.orient.ox.ac.uk/>. Acceso en: $21 / 08 / 2016$.
} 
En cuanto a la alusión a los mar-tu en la literatura mesopotámica, tenemos varias referencias; la más importante, El casamiento de Martu. En la narración, Martu está decidido a buscar una compañera para casarse dado que debe pagar una tributación-ración igual a la de un hombre casado, pero estando él todavía soltero. Por otra parte, el relato ofrece una caracterización opuesta de las personas que viven por fuera de la ciudad frente a las que viven en ella. En diferentes instancias, al grupo poblacional al que pertenece Martu se lo describe con hábitos contrarios a los ideales urbanos, puesto que come carne cruda, deambula de un lado a otro, no conoce los lugares de adoración, vive en tiendas, etc. Veamos cómo se presenta la cuestión en el relato:

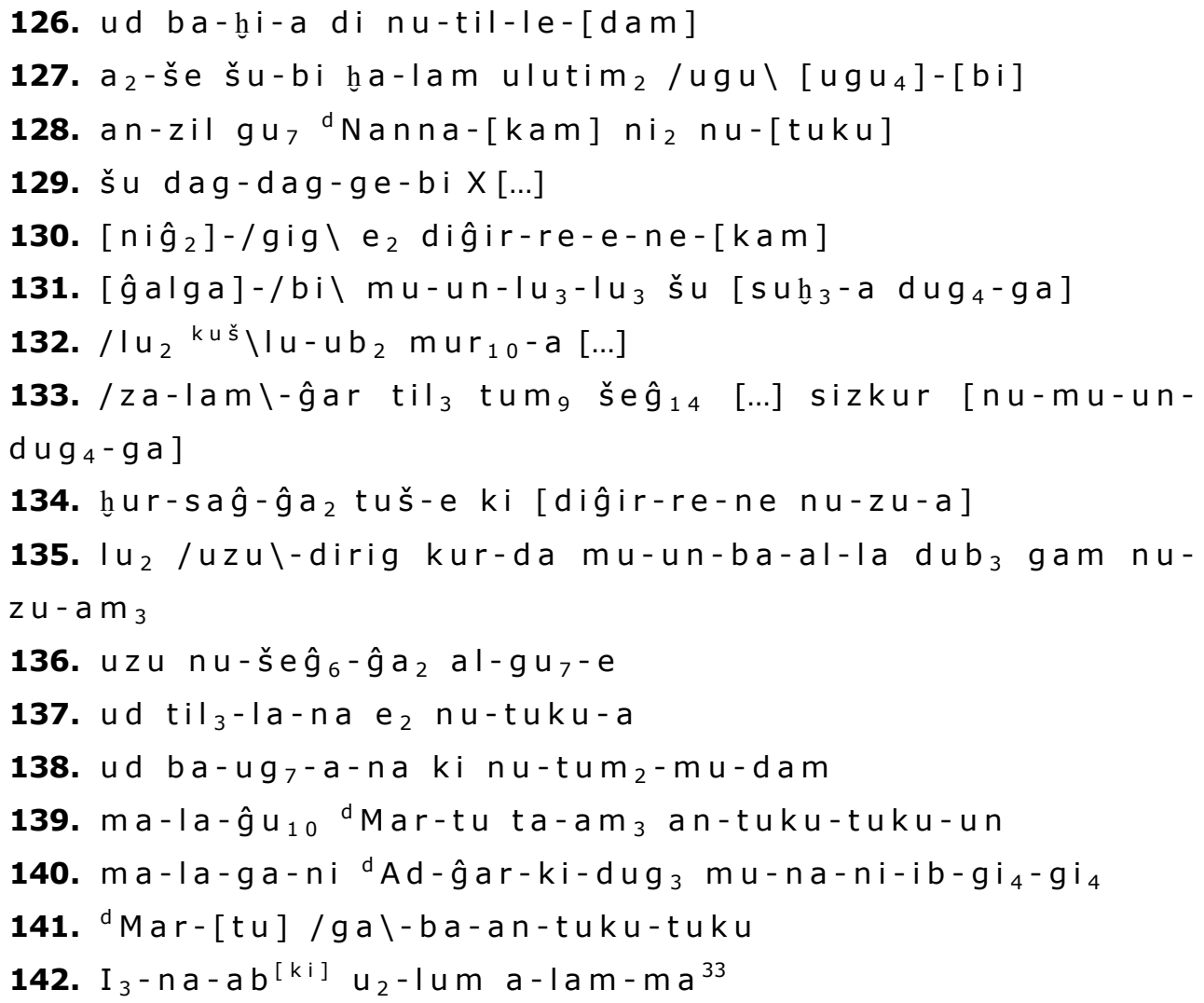

[126. Los días se multiplicaron, no se tomó ninguna decisión (La amiga de Adĝarkidug le dice a ella):

127. "Ahora, sus manos son destructivas y su forma es como la de un mono,

128. Ios tabúes alimenticios de Nanna come y no tiene miedo,

129. no para de vagar a través de $[\ldots]$,

130. es una abominación a los templos de los dioses,

131. sus razones mezcla, las transforma en una confusión,

132. se viste con un saco de cuero $[\ldots]$,

133. vive en tiendas (expuesto al) viento y la lluvia $[. .$.$] no pronuncia plegarias,$

134. se asienta en las montañas, desconoce los sitios de los dioses,

135. desentierra hongos de la montaña, no sabe cómo inclinarse,

136. come carne cruda,

${ }^{33}$ BLACK, Jeremy et al. The Electronic Text Corpus... Op. cit., c.1.7.1 y t.1.7.1. 
137. no tiene casa durante su vida,

138. cuando muere no debe ser llevado a un sitio de enterramiento.

139. Amiga mía, ¿te casarás con Martu?"

140. A su amiga, Adĝar-kidug le responde:

141. "iMe casaré con Martu!".

142. iInab, ulum, alam!]

En la descripción del universo de Martu, hay una apreciación negativa de sus costumbres, ya que se le atribuyen cualidades inferiores frente a los habitantes de las ciudades. Este imaginario de la población mar-tu/amorrea se encuentra en otros textos como la Lista de Reyes Asirios, ${ }^{34}$ en la cual, se alude a los diecisiete primeros gobernantes, los cuales "vivieron en tiendas" (a-ši-bu-tu kul-ta-re), y se sostiene, de este modo, que los primeros monarcas no residían en la ciudad de Aššur. ${ }^{35}$ Sin embargo, la asociación con prácticas de vida semi-nómade no poseía las connotaciones negativas que adquieren en algunos textos literarios como El casamiento de Martu. Además, la descripción que encontramos en la Lista de Reyes Asirios de sus gobernantes puede enlazarse con la infiltración de población amorrea en la región, sobre todo en la parte septentrional de Mesopotamia así como en la Siria occidental y el Éufrates central, donde se señala la presencia de tribus de pastores trashumantes haneas como los Yaminitas y Sim'alitas, algunas controlando la ciudad de Mari. ${ }^{36}$

Asimismo, con el término mar-tu se nombra en textos literarios como Enki y el orden mundo, Lugalbanda y el pájaro Anzu y La maldición de Akkad a ciertas poblaciones caracterizadas como nómades, que desconocen la agricultura y que tampoco viven en ciudades. Esta construcción peyorativa y obviamente ficcional tiende a mostrar una realidad exagerada y hasta alterada, puesto que no hay registros de una infiltración violenta de lo amorreos en Mesopotamia, sino que fueron aculturándose y apoderándose de las tradiciones sumerias y acadias.

Como contracara de esta imagen negativa, en dos himnos reales de Šulgi de Ur (B y C), el monarca se jacta de ser políglota $\mathrm{y}$, además, de poder hablar la lengua de los mar-tu . Veamos qué sucede en Šulgi B:

213. 4-kam-ma-aš [mar-tu]luz kur-ra $A[X(X)]$

214. eme bal-e mu-un-da-an-gub-be ${ }_{2}{ }^{37}$

[213. Cuarto: puedo ser de un mar-tu, un hombre de las montañas,

\footnotetext{
${ }^{34}$ Cf. POEBEL, Arno. The Assyrian King List from Khorsabad. Journal of Near Eastern Studies, v.1, n. 3, 1942, p. 247-306; POEBEL, Arno. The Assyrian King List from Khorsabad (Continued). Journal of Near Eastern Studies, v. 1, n. 4, 1942, p. 460-492; POEBEL, Arno. The Assyrian King List from Khorsabad (Concluded). Journal of Near Eastern Studies, v. 2, n. 1,1943, p. 56-90; GELB, Ignace. Two Assyrian King Lists. Journal of Near Eastern Studies, v. 13, n. 4,1954, p. 209-230.

35 POEBEL, Arno. The Assyrian King List... Op. cit., p. 252.

${ }^{36}$ WEISS, Harvey. Seventeen Kings Who Lived in Tents. En: HÖFLMAYER, Felix. (ed.). The Late Third Millennium in the Ancient Near East Chronology, C14, and Climate Change. Papers from the Oriental Institute Seminar, The Early/Middle Bronze Age Transition in the Ancient Near East: Chronology, C14, and Climate Change, held at the Oriental Institute of the University of Chicago 7-8 March 2014. Chicago: The University of Chicago Press, 2017, p. 145.

${ }^{37}$ BLACK, Jeremy et al. The Electronic Text Corpus... Op. cit., c.2.4.2.02 y t.2.4.2.02.
} 
214. el traductor de su lengua.]

En el Cilindro de Ciro (líneas 29 y 30), una narración neo-babilónica encomiástica del monarca persa, se retoma la descripción de los amorreos semejante a la que aparece en El casamiento de Martu: "(...) aquellos que habitan [remotos distri]tos (y) los reyes de la tierra de Amurru que viven en tiendas, todos ellos, trajeron sus pesados tributos a Shuanna, y besaron mis pies". ${ }^{38}$

De alguna manera, la recurrencia a ciertos arquetipos poblacionales en la literatura mesopotámica, que exhiben rasgos considerados inferiores, tienen a los mar-tu como uno de sus principales referentes. Sin embargo, la apreciación negativa se transforma cuando se los señala en los textos administrativo-económicos, puesto que ahí se los puede ver interactuar e interferir ineludible y cotidianamente en la economía mesopotámica. Sin embargo, el cruce no habría apuntado únicamente a la esfera económica, sino también a la política y la cultural.

Con este horizonte más amplio en mente, podemos sugerir que los cambios introducidos en el uso del sumerio como lengua escrita, en cuanto a su estructura sintáctica y semántica, comenzó a sufrir modificaciones no sólo bajo la influencia y desarrollo paralelo del acadio en cuanto lengua semita, sino también de otras poblaciones cercanas. En cierto modo, durante el período paleo-babilónico, cuando el sumerio se mantuvo como lengua litúrgica por parte de las dinastías de Isin y Larsa, fue lentamente perdiendo poder y preponderancia bajo el influjo del acadio, con el que los nuevos reyes amorreos se sentían más emparentados en la esfera lingüística. Curiosamente, el acadio se transformó en la lengua hegemónica en todos los ámbitos discursivos sobre todo a partir del predominio de la ciudad de Babilonia. No obstante, en las escuelas de escribas, el sumerio seguía aprendiéndose, pero sufriría la influencia y las modificaciones que los hablantes de una lengua semita le imprimirían.

\section{Palabras finales}

El bilingüismo sumerio-acadio se habría prolongado desde el Dinástico Temprano hasta el período paleo-babilónico, cuando se produjo la toma del poder en la Baja Mesopotamia por una serie de dinastías de origen amorreo ( $\mathrm{mar}-\mathrm{tu}$ ). En esa amplia franja temporal, que va del 2900 al 1600 a.C. de manera aproximada, el sumerio y el acadio, aunque no estaban emparentados lingüísticamente, se influenciaron de forma recíproca provocando cambios en ambas lenguas.

En paralelo a la afluencia de préstamos lingüísticos entre ambas lenguas, los ma r- tu en época paleo-babilónica abandonaron paulatinamente la utilización del sumerio en textos administrativos, legales y literarios, haciéndose extensivo el acadio. En términos comparativos,

\footnotetext{
${ }^{38}$ Cf. Cyrus Cylinder. Disponible en:

<http://www.britishmuseum.org/explore/highlights/articles/c/cyrus_cylinder_-_translation.aspx>. Acceso en: 21/08/2016.
} 
para el reinado de Hammurabi de Babilonia el empleo del sumerio se vuelve escaso si lo contrastamos con el uso dado en las dinastías de Isin y/o Larsa.

Por otro lado, para la historia del Próximo Oriente antiguo, es crucial considerar los datos suministrados tanto por el registro arqueológico como el epigráfico en cuanto unidad de análisis, puesto que la información que en muchas ocasiones se puntualiza en los manuscritos forma parte de un constructo ideológico afín a un proceso histórico y no debe ser abordada de forma independiente. Por consiguiente, las descripciones pseudo-etnográficas, que se encuentran, por ejemplo, en El casamiento de Martu, corresponden más bien a una construcción ficcional más que a una fotografía del momento.

Rodrigo Cabrera: Doutorando em História pela Facultad de Filosofía y Letras da Universidad de Buenos Aires com o projeto de pesquisa "Espacios consagrados para la muerte y rituales conmemorativos. La depositación de ofrendas funerarias en Súmer (entre el Dinástico Temprano IIIb y el período neo-sumerio, c. 2540-2004 a.C.)". E também Licenciado em História (equivalente ao Mestrado) pela mesma universidade e Professor da História Antiga I (Oriente) e seminários de línguas orientais (sumério). Bolsista no Consejo Nacional de Investigaciones Científicas y Técnicas (CONICET, Argentina) com local de trabalho no Instituto Multidisciplinario de Historia y Ciencias Humanas (IMHICIHU). 